\title{
Diversity Multiplexing Tradeoff in Multiple Antenna Multiple Access Channels with Partial CSIT
}

\author{
Kaushik Josiam, Dinesh Rajan and Mandyam Srinath, \\ Department of Electrical Engineering, Southern Methodist University, \\ Dallas, TX 75275, USA, Email: \{kjosiam, rajand, mds\}@engr.smu.edu.
}

\begin{abstract}
We derive a lower bound on the diversitymultiplexing tradeoff for multiple antenna multiple access channels using temporal power control (PC). We quantify the substantial improvements in diversity gains with finite rate feedback over schemes that use no feedback, even when users have asymmetric diversity and rate requirements. For small multiplexing gains, all users in the MAC achieve their single user performance as if each receives $B$ distinct bits of feedback. We also show that for a SISO MAC with finite rate feedback, the diversity orders with PC and with opportunistic user selection are the same at all multiplexing gains.
\end{abstract}

\section{INTRODUCTION}

The benefits of channel state information at the transmitter (CSIT) have been established for both point-to-point and multi-user fading channels. For a point-to-point link, perfect CSIT can improve diversity [1], outage [2] and channel capacity [3]. In particular, with perfect CSIT, the improvement in diversity is shown to be exponential [1]. Since perfect CSIT is not practical, a finite rate feedback of CSI to the transmitter is widely used in practice. In this paper, we use the diversitymultiplexing (D-MG) tradeoff [4] framework to quantify the performance improvements with partial CSIT in a multiple access channel (MAC) . With partial CSIT, the diversity gains differ largely on the strategy used by the transmitter. The DMG tradeoffs for a point-to-point link with partial CSIT, have been characterized when the transmitter uses beamforming [5] and power control (PC) [6]. In multiuser environments, the D-MG tradeoff with partial CSIT has been derived for a broadcast channel when the transmitter uses the feedback for scheduling and beamforming [7] and for MAC, broadcast and relay channels in [8], when the feedback is used for opportunistic user selection.

In this paper, we characterize the D-MG tradeoff for a multiple-input, multiple-output (MIMO) MAC when partial CSIT is used to perform PC across all users. The receiver is assumed to have perfect channel knowledge. The partial CSIT in the form of $B$ bits of feedback represents the quantized information about the channel. We build on the work in [6], where the authors characterized the D-MG tradeoff for a pointto-point MIMO link with PC. The derived tradeoff is a lower bound, since the optimal D-MG tradeoff for a MAC with partial CSIT is still unknown. The improvement in diversity due to PC is promised even when users have asymmetric diversity and rate requirements and the maximum diversity

This work has been supported in part by NSF under grant CCF 0546519. possible is not limited by the user with the minimum diversity requirement. When all users in the MAC transmit at the same rate, we show that at smaller multiplexing gains, each user can achieve his single-user performance with emphatic improvements in diversity with just one bit feedback. The diversity improvements are as if each user received a distinct feedback codeword, when in reality just one generic codeword was broadcast to all users. Finally, for a SISO MAC, we show that the D-MG tradeoff with power control coincides with the tradeoff with opportunistic user selection [8]. This D-MG equivalence is despite qualitative differences in the nature of feedback: for power control, we quantize the actual channel realization itself, while the feedback codeword with opportunistic user selection indicates the chosen user.

The rest of the paper is structured as follows: The system set-up and the problem formulation are described in Sec II. Related work is explained in Sec III. Our main result for the MAC is derived in Sec IV and illustrated in Sec V. We end with a summary in Sec VI.

Notation: Uppercase boldface letters represent matrices. $\mathbf{H}^{\dagger}, \operatorname{det}(\mathbf{H})$ and $\operatorname{tr}(\mathbf{H})$ represent the hermitian transpose, determinant and the trace of matrix $\mathbf{H} . \mathbb{C N}(0,1)$ denotes a complex Gaussian random variable with zero mean and unit variance. The notation, $\doteq$, in $f(\beta) \doteq \beta^{a}$ denotes asymptotic equality [4]. $\dot{\leq}, \geq$ are similarly defined.

\section{System Model and Problem Formulation}

Consider a MIMO MAC with a feedback link from the base station (BS) to the $K$ users as shown in Fig. 1. The wireless link between user $i$ and the BS is described by a single flat fading path between each of its $m_{i}$ transmit and $n$ receive antennas. The channel gains between all pairs of transmit and receive antennas for the $i^{t h}$ user are grouped in a $n \times m_{i}$-dimensional channel matrix, $\mathbf{H}_{i}$, whose elements are assumed to be i.i.d. $\mathbb{C N}(0,1)$. Further, the channel is assumed to be block fading i.e., the entries in the channel matrix change independently after coherence time, $t_{c}$ symbols. At the beginning of a coherence interval, the receiver sends a feedback codeword $Q(\{\mathbf{H}\})$, to the transmitter, where $Q: \mathbb{C}^{n \times \sum_{i} m_{i}} \rightarrow\{1, \ldots, L\}$ and $\mathbf{H}=\left[\mathbf{H}_{1}, \ldots, \mathbf{H}_{K}\right]$ is formed by concatenating channel matrices from all users. The feedback codeword is assumed to be available to the users instantaneously, without errors and indicates the transmission scheme that the users must use to transmit the data in that coherence interval. 


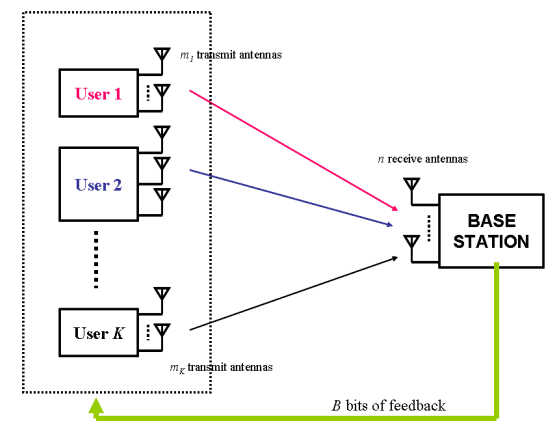

Fig. 1. A MIMO MAC with $B$ bits of feedback

In this paper, we focus on transmission schemes that use temporal PC. Due to limited number of feedback bits available, we consider a scheme where the same transmit power is used at all antennas, by all users. Specifically, the feedback codeword indicates the power, $P_{Q}(\mathbf{H})$, at each of the transmit antennas in the MAC. Let $\mathbf{X}_{i}$ be the $m_{i} \times t_{c}$-dimensional complex transmit matrix for the $i^{t h}$ user. Then, the $n \times t_{c}$ dimensional received matrix, $\mathbf{Y}$, at the $\mathrm{BS}$ is given by,

$$
\mathbf{Y}=\sqrt{P_{Q}(\mathbf{H})} \sum_{i=1}^{K} \mathbf{H}_{i} \mathbf{X}_{i}+\mathbf{Z},
$$

where $\mathbf{Z}$ is the $n \times t_{c}$-dimensional additive white Gaussian noise - $\mathbb{C N}(0,1)$. To transmit at power $P_{Q}(\mathbf{H})$, each codeword $\mathbf{X}_{i}$ must satisfy the constraint, $\operatorname{tr}\left\{\mathbb{E}\left[\mathbf{X}_{i} \mathbf{X}_{i}^{\dagger}\right]\right\}=m_{i} t_{c}$. Without loss of optimality, we assume that the transmit codeword, $\mathbf{X}_{i}$, for user $i$ is generated as i.i.d. $\mathbb{C N}(0,1)$ i.e., $\mathbb{E}\left[\mathbf{X}_{i} \mathbf{X}_{i}^{\dagger}\right]=I_{m}$. Additionally, we impose a long term power constraint on the transmitted signals from every transmit antenna in the MAC, $\lim _{T \rightarrow \infty} \frac{1}{T} \sum_{t=1}^{T}\left[P_{Q}(\mathbf{H}(t))\right] \leq P_{a v}$, where $\mathbf{H}(t)$ is the concatenated channel at time $t$. This equal average power constraint per antenna simplifies the computation of the DMG tradeoff and translates to users having different average powers i.e., $P_{a v}^{i}=m_{i} P_{a v} \forall i$. However, the asymptotic DMG tradeoff can be computed even when this equal average power constraint is relaxed using the same procedure as in [9], [10]. We briefly discuss the unequal average power per antenna case in Sec. IV.

The spatial multiplexing gain, $r_{k}$, for user $k$ is defined as $r_{k}=\lim _{P_{a v} \rightarrow \infty} \frac{R_{k}\left(P_{a v}\right)}{\log \left(P_{a v}\right)}$, where $R_{k}$ is its rate of transmission. For a given achievable $K$-tuple of multiplexing gains $\left(r_{1}, \ldots, r_{K}\right)$, the diversity order $d_{k}^{p c}$ for user, $k$ is defined as, $d_{k}^{p c}\left(r_{1}, \ldots, r_{K}\right)=\lim _{P_{a v} \rightarrow \infty}-\frac{\log \left(\operatorname{Pr}^{(k)}\left(P_{a v}\right)\right)}{\log \left(P_{a v}\right)}$, where $\left.\operatorname{Pr}^{(k)}\left(P_{a v}\right)\right)$ is its probability of error. For each rate-tuple, define $d_{k}^{p c}\left(r_{1}, \ldots, r_{K}\right)$ as the supremum of the diversity gains for the $k^{\text {th }}$ user over all coding schemes.

\section{QUANTIZED FEEDBACK IN MIMO CHANNELS}

This section is a summary of the D-MG tradeoff for pointto-point MIMO link with finite rate feedback [6]. For a MIMO Rayleigh fading channel, $\mathbf{H} \in \mathbb{C}^{n \times m}$, the outage probability completely depends on the $\min \{m, n\}$ eigenvalues of the Wishart matrix, $\mathbf{H H}^{\dagger}$ [11]. With finite feedback resource, the designer of the quantizer of the $\min \{m, n\}$ eigenvalues that

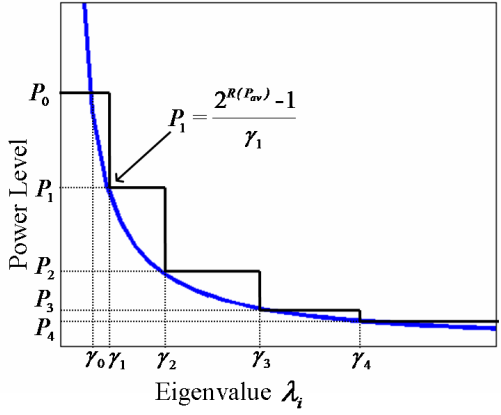

Fig. 2. Ideal and quantized inversion power allocation [6].

minimizes the outage probability is critical. The asymptotic optimality of a scalar equi-power quantizer that quantizes just one of the eigenvalues of $\mathbf{H H}^{\dagger}$ is shown in [6]. Since, we use this equi-power quantizer for the MAC, we now briefly outline its design.

Given $\lambda_{i}$, an eigenvalue of $\mathbf{H H}^{\dagger}$, the ideal power allocation that minimizes the outage probability is the inversion power allocation shown in Fig. 2. Also shown is the piecewise step function that is the quantized approximation to the ideal power allocation curve. The inversion power allocation ensures outage free transmission at rate $R\left(P_{a v}\right)$ for the interval $\left[\gamma_{j}, \gamma_{j+1}\right)$, by specifying power $P_{j}=\frac{2^{R\left(P_{a v}\right)}-1}{\gamma_{j}}$. If $\lambda_{i} \in\left[\gamma_{j}, \gamma_{j+1}\right)$, then the index of the interval is sent to the transmitter which then uses power $P_{j}$ to transmit from all antennas. When $\lambda_{i} \rightarrow 0$, inversion power allocation requires infinite power to minimize outage probability. However, due to finite average power constraints, allocating infinite power is not possible. Therefore, for the first quantization bin, $\left[0, \gamma_{1}\right)$, the power $P_{0}$ is assigned so that the average power constraint is satisfied. If $\lambda_{i}<\gamma_{1}$, outage free communication is guaranteed only for $\lambda_{i}>\gamma_{0}$, where $\gamma_{0}$ is defined as, $\gamma_{0}=\frac{2^{R(P a v)}-1}{P_{0}}$. For $\lambda_{i}<\gamma_{0}, P_{0}$ is insufficient to prevent outage, and consequently, the outage probability, $\operatorname{Pr}^{\mathcal{O}}\left(R\left(P_{a v}\right)\right)$, is defined as, $\operatorname{Pr}^{\mathcal{O}}\left(R\left(P_{a v}\right)\right)=\operatorname{Pr}\left[\lambda_{i}<\gamma_{0}\right]$. The quantization intervals, $\left\{\gamma_{i}\right\}$ and the allocated power, $P_{Q}\left(\lambda_{i}\right)$ are defined using the scalar equi-power quantizer. The probability density function (pdf) is known for the eigenvalues of a Wishart matrix. Hence, given pdf, $f_{\lambda_{i}}(x)$, of the eigenvalue $\lambda_{i}$, the average power is computed as,

$$
P_{a v}=P_{0} F_{\lambda_{i}}\left(0, \gamma_{1}\right)+\cdots+P_{L-1} F_{\lambda_{i}}\left(\gamma_{L-1}, \infty\right),
$$

where $F_{\lambda_{i}}(a, b)=\int_{a}^{b} f_{\lambda_{i}}(x) d x$ is the probability mass concentrated in the interval $[a, b]$. To compute $\left\{\gamma_{j}\right\}_{j}$, the equipower quantizer allocates equal power to each quantizing bin,

$$
P_{0} F_{\lambda_{i}}\left(0, \gamma_{1}\right)=P_{j} F_{\lambda_{i}}\left(\gamma_{j}, \gamma_{j+1}\right) \cdots=P_{L-1} F_{\lambda_{i}}\left(\gamma_{L-1}, \infty\right) .
$$

Combining (2) and (3),

$$
P_{j} F_{\lambda_{i}}\left(\gamma_{j}, \gamma_{j+1}\right)=P_{a v} / L, \quad \forall j \in 0,1, \ldots, L-1 .
$$

From (4), when $j=L-1$,

$$
P_{L-1} F_{\lambda_{i}}\left(\gamma_{L-1}, \infty\right)=P_{a v} / L
$$


Since, $P_{L-1}=\left(2^{R\left(P_{a v}\right)}-1\right) / \gamma_{L-1}$, (5) is a function in $P_{L-1}$ (or $\left.\gamma_{L-1}\right)$. Hence, $P_{L-1}$ and $\gamma_{L-1}$ are computed using (5). The value of $\gamma_{L-1}^{*}$ is then used in (4) for $j=L-2$ to compute both $P_{L-2}$ and $\gamma_{L-2}^{*}$ and the process is iterated over all $L$ intervals until all $P_{j} \forall j$ are computed.

$D-M G$ Tradeoff: The quantized eigenvalue from $\mathbf{H H}^{\dagger}$ should maximize the achievable diversity associated with the outage probability for a given multiplexing gain. To achieve a multiplexing gain of $r$, the strategy is to use at least $i=\lceil r\rceil$ of the largest eigenvalues and ensure that the allocated power is sufficient to support a multiplexing gain of one on the $i^{\text {th }}$ largest eigenvalue, $\lambda_{i}$. There are $(i-1)$ eigenvalues that are greater than $\lambda_{i}$ and thus, a total multiplexing gain of $r$ is guaranteed from the largest $i$ eigenvalues. The actual eigenvalue that is quantized could be any of the remaining $(\min \{m, n\}-i+1)$ smaller eigenvalues since they all can support the required rate; among these eigenvalue the one that maximizes diversity for the given rate is selected. The D-MG tradeoff in the limit $P_{a v} \rightarrow \infty$, is stated from [6] in the following theorem.

Theorem 1: In a point-to-point MIMO link, with $m$ transmit, $n$ receive antennas $(m \leq n)$ and a sufficiently large coherence time $t_{c}$, when a scalar quantizer with $L$ bins is used at the receiver, the diversity order, $d_{m, n, L}^{p c}$, at multiplexing gain $r$ is given by,

$$
\begin{aligned}
d_{m, n, L}^{p c}(r)= & \max _{i \in\{\lceil r\rceil, \ldots, m\}}[(1-r / i) \\
(n-\lceil r\rceil+1) & (m-\lceil r\rceil+1) G(m, n, i, l)],
\end{aligned}
$$

where $G(m, n, i, l) \triangleq \sum_{l=0}^{L-1}[(n-i+1)(m-i+1)]^{l}$. Next, we use this equi-power quantizer to derive a lower bound on the achievable D-MG tradeoff in a MAC with PC.

\section{D-MG TRAdeoff IN A MAC With QuAntized PC}

The capacity region of a MAC is defined by a set of $2^{K}-1$ inequalities on the transmission rates; one inequality for each non-empty subset, $S \subseteq\{1,2, \ldots, K\}$, of users. The feedback information and transmit power is selected to meet not only the individual rates for each user, but also the various sum rate requirements.

Consider an arbitrary subset, $S$, of users who are in outage and whose target rate is $\sum_{i \in S} r_{i}$. Since the users in $S^{c}$ are not in outage, the receiver can recover their data symbols, $\mathbf{X}_{S^{c}}$, without any errors. Therefore, the receiver can remove contribution of $\mathbf{X}_{S^{c}}$ from the received signal, $\mathbf{Y}$ in (1) and the updated received signal is expressed as,

$$
\mathbf{Y}_{S}=\sqrt{P_{Q}(\mathbf{H})} \sum_{i \in S} \mathbf{H}_{i} \mathbf{X}_{i}+\mathbf{Z}=\sqrt{P_{Q}(\mathbf{H})} \mathbf{H}_{S} \mathbf{X}_{S}+\mathbf{Z}
$$

where $\mathbf{H}_{S} \in \mathbb{C}^{n \times \sum_{i \in S} m_{i}}$ and $\mathbf{X}_{S} \in \mathbb{C}^{\sum_{i \in S} m_{i} \times t_{c}}$ are the concatenated channel and transmit symbol matrices respectively for the users in $S$. Thus, (7) is equivalent to a point-to-point link with $\sum_{i \in S} m_{i}$ transmit and $n$ receive antennas. The ordered eigenvalues of the Wishart matrix, $\mathbf{H}_{S} \mathbf{H}_{S}^{\dagger}$, is given by,

$$
\lambda_{1}^{(S)} \leq \lambda_{2}^{(S)} \leq \cdots \leq \lambda_{\min \left\{\sum_{i \in S} m_{i}, n\right\}}^{(S)}
$$

We now follow the same principle of eigenvalue selection as the point-to-point link [6] described in Sec III. Therefore, to minimize outage for users in set $S$, when the target rate required is $\sum_{i \in S} r_{i}$, we select an eigenvalue from (8) whose index maximizes (6). Define $j_{S}^{*}$ as the index of the eigenvalue that minimizes outage for the set, $S$, i.e.,

$$
\begin{aligned}
& j_{S}^{*}=\max _{j=\left\{\left\lceil\sum_{i \in S} r_{i}\right\rceil, \ldots, \min \left\{\sum_{i \in S} m_{i}, n\right\}\right\}}\left\{\left(1-\sum_{i \in S} r_{i} / j\right)\right. \\
& \left.\left(n-\left\lceil\sum_{i \in S} r_{i}\right\rceil+1\right)\left(\sum_{i \in S} m_{i}-\left\lceil\sum_{i \in S} r_{i}\right\rceil+1\right) G\left(\sum_{i \in S} m_{i}, n, j, l\right)\right\} .
\end{aligned}
$$

We collect these outage minimizing eigenvalues, that also guarantee the associated sum-rate requirements from all $2^{K}-$ 1 subsets into the set, $\Lambda\left(r_{1}, \ldots, r_{K}\right)=\left\{\lambda_{j_{S}^{*}}^{(S)}: \forall S \subseteq\right.$ $\{1, \ldots, K\}\}$. Due to finite rate feedback, we choose to quantize one eigenvalue from the set $\Lambda\left(r_{1}, \ldots, r_{K}\right)$. Since the quantizer is designed based on a channel inversion PC, the smallest eigenvalue is associated with the most transmit power. Therefore, to ensure that the transmit power specified by the feedback is sufficient to satisfy all target sum-rate requirements in the MAC, we quantize the minimum eigenvalue in the set $\Lambda\left(r_{1}, \ldots, r_{K}\right)$ using the scalar equi-power quantizer with $L$ levels. Thus, the transmit power for all users in the MAC is determined by the quantization of a single eigenvalue. However at small multiplexing gains, we will show that the effect of such quantization is magnified, i.e., it is as if each user's feedback was a distinct quantization codeword. Define $S^{*}=\arg \min _{S} \lambda_{j_{S}^{*}}^{(S)}$, as the subset of users, whose concatenated channel matrix contained the minimum eigenvalue of $\Lambda\left(r_{1}, \ldots, r_{K}\right)$. We now define outage probability per user to characterize the D-MG tradeoff.

Definition: For a MAC with $K$ users, given a rate vector, $\left(R_{1}\left(P_{a v}\right), \ldots, R_{K}\left(P_{a v}\right)\right)$, the event that the $k^{t h}$ user is in outage is $\mathcal{O}_{k} \triangleq \bigcup_{S_{k}} \mathcal{O}_{S_{k}}$, where the union is over all subsets $S_{k}=\left\{\{k\} \cup \tilde{S}_{k}, \forall \tilde{S}_{k} \subseteq\{1,2, \ldots, k-1, k+1, \ldots, K\}\right\}$ containing the user $k$. The outage event $\mathcal{O}_{S_{k}}$ for a subset $S_{k}$, represents the event when the eigenvalue of $\mathbf{H}_{S_{k}} \mathbf{H}_{S_{k}}^{\dagger}$ chosen for quantization is so small it falls below the channel inversion threshold, $\gamma_{0_{j_{S_{k}}}}$, defined by the quantizer i.e.,

$$
\mathcal{O}_{S_{k}} \triangleq\left\{\lambda_{\left.\Gamma \sum_{i \in S_{k}}^{\left(S_{k}\right)} r_{i}\right\rceil}<\gamma_{0_{j_{S_{k}}}}\right\}
$$

where $j_{S_{k}} \in\left\{\left\lceil\sum_{i \in S_{k}} r_{i}\right\rceil, \ldots, \min \left\{\sum_{i \in S_{k}} m_{i}, n\right\}\right\}$ is the index of the eigenvalues that can be quantized to meet the desired rate.

Thus, the outage event for a user subset involving the $k^{t h}$ user, $\mathcal{O}_{S_{k}}$, in the MAC given in (10) evaluates the outage probability, as if the eigenvalue quantized in the MAC belongs to the concatenated channel matrix of set $S_{k}$, i.e., $S_{k}=S^{*}$. But, when $S_{k} \neq S^{*}$, the users in the subset $S_{k}$ have lower outage probability. Hence, the definition of outage using $S_{k}$ represents the worst-case outage probability for user $k$ in the MAC and thus characterizes the limiting diversity for that user. The outage probability and the consequent diversity order for the $k^{t h}$ user in a MAC are derived in Theorem 2. 
Theorem 2: Consider a MAC with $K$ users, where the $i^{\text {th }}$ user has $m_{i}$ transmit antennas and the $\mathrm{BS}$ has $n$ receive antennas. Let a scalar quantizer with $L$ bins be used at the BS. For large block length, $t_{c}$, the maximum achievable diversity order, $d_{k}^{p c}\left(r_{1}, \ldots, r_{K}\right)$, for any user $k$ in the MAC, given a achievable rate vector $\left(r_{1}, \ldots, r_{K}\right)$ is described as,

$$
d_{k}^{p c}\left(r_{1}, \ldots, r_{K}\right)=\min _{S_{k}} d^{p c} \sum_{i \in S_{k}} m_{i}, n, L\left(\sum_{i \in S_{k}} r_{i}\right),
$$

where $S_{k}$ is the subset of users containing user $k, d_{m, n, L}^{p c}(r)$ is the diversity order defined in (6) for a point-to-point link with $m$ transmit, $n$ receive antennas and PC with $L$ bin quantizer.

Proof: To compute $\mathcal{O}_{S_{k}}$, we assume that the users in $S_{k}^{c}$ are not in outage and their transmit symbols $\mathbf{X}_{S_{k}^{c}}$ can be decoded by the receiver without errors. Therefore, the receiver cancels the contribution of $\mathbf{X}_{S_{k}^{c}}$ from the received signal in (1). By replacing $S$ with $S_{k}$ in (7), we see that the system is now reduced to a point-to-point link with $\sum_{i \in S_{k}} m_{i}$ transmit and $n$ receive antennas, and a fading matrix $\mathbf{H}_{S_{k}}$ whose entries are i.i.d. complex Gaussian. Let the desired data rate for user $i$ be, $R_{i}\left(P_{a v}\right)=r_{i} \log P_{a v}$. Then the outage probability for the users in $S_{k}$ is computed using [6] as,

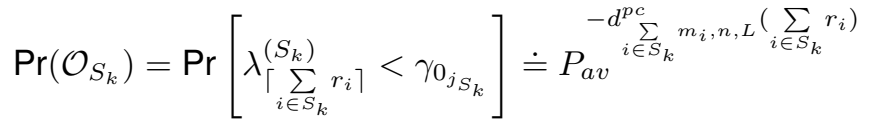

Therefore, the probability that user $k$ is in outage is given by,

$$
\operatorname{Pr}\left(\mathcal{O}_{k}\right)=\operatorname{Pr}\left(\bigcup_{S_{k}} \mathcal{O}_{S_{k}}\right) \leq \sum_{S_{k}} \operatorname{Pr}\left(\mathcal{O}_{S_{k}}\right) \doteq \operatorname{Pr}\left(\mathcal{O}_{S_{k}^{*}}\right) .
$$

where $S_{k}^{*}$ is the subset with the slowest decay rate of $\operatorname{Pr}\left(\mathcal{O}_{S_{k}}\right)$,

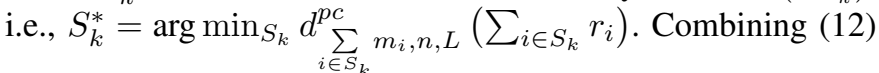
with the fact that $\operatorname{Pr}\left(\mathcal{O}_{k}\right) \geq \operatorname{Pr}\left(\mathcal{O}_{S_{k}^{*}}\right)$, we have

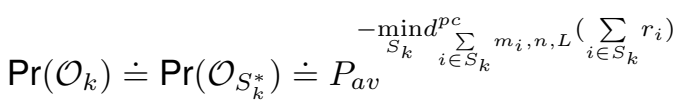

The probability of error for the $k^{t h}$ user, $\operatorname{Pr}^{(k)}\left(P_{a v}\right)$ is bounded as [4],

$$
\operatorname{Pr}\left(\mathcal{O}_{k}\right) \leq \operatorname{Pr}^{(k)}\left(P_{a v}\right) \leq \operatorname{Pr}\left(\mathcal{O}_{k}\right)+\operatorname{Pr}^{(k)}(\text { no outage }) .
$$

To compute the probability of error under no outage, let the eigenvalue chosen for quantization support a rate $r_{S_{k}}^{*}$ that is larger than the desired rate $\sum_{i \in S_{k}} r_{i}$ for the set of users in $S_{k}$ i.e., $\log \operatorname{det}\left(I+P_{Q}(\mathbf{H}) \mathbf{H}_{S_{k}} \mathbf{H}_{S_{k}}^{\dagger}\right)=r_{S_{k}}^{*} \log \left(P_{a v}\right)$. Then, using a typical set decoder as in [10], we can compute the probability of error under no outage,

$$
\begin{aligned}
\operatorname{Pr}^{(k)}(\text { no outage }) & \leq \sum_{S_{k}} P_{a v}^{t_{c} \sum_{i \in S_{k}} r_{i}} \operatorname{det}\left(I+P_{Q}(\mathbf{H}) \mathbf{H}_{S_{k}} \mathbf{H}_{S_{k}}^{\dagger}\right)^{-t_{c}} \\
& =\sum_{S_{k}} P_{a v}^{t_{c} \sum_{i \in S_{k}} r_{i}-t_{c} r_{S_{k}}^{*}} \stackrel{(a)}{=} P_{a v}^{-\min _{S_{k}} t_{c} \delta_{S_{k}}}
\end{aligned}
$$

where $(a)$ is due to substituting $r^{*}=\sum_{i \in S_{k}} r_{i}+\delta_{S_{k}}$ and $\delta_{S_{k}}>0$ is the excess rate beyond the target rate. For a large enough $t_{c}, \min _{S_{k}} t_{c} \delta_{S_{k}}$ is larger than the exponent of the outage probability given in (13). Since the diversity order is determined by the term with the smaller exponent, the upper bound on the probability of error in (14) is dominated by the outage probability. Therefore, the upper and lower bounds on the probability of error in (14) match and consequently, the maximal diversity with $\mathrm{PC}$ is given by (11).

The above derivation was based on the assumption of identical average power constraints at all transmit antennas. But consider a scenario where, instead of average power constraint per antenna, the constraint requires that the transmit power per user be identical. Such a constraint would scale the instantaneous power level, $P_{Q}(\mathbf{H})$ equally at all antennas and the received signal in (1) can be written as,

$\mathbf{Y}=\sum_{i=1}^{K} \sqrt{P_{Q}(\mathbf{H}) / m_{i}} \mathbf{H}_{i} \mathbf{X}_{i}+\mathbf{Z}=\sqrt{P_{Q}(\mathbf{H})} \mathbf{H}_{K} \mathbf{X}_{K}+\mathbf{Z}$,

where $\mathbf{H}_{K} \in \mathbb{C}^{n \times \sum_{i} m_{i}}$ is the concatenated channel matrix of the $K$ users with entries $\left\{\sqrt{1 / m_{i}} \mathbf{H}_{i}\right\}_{i}$ that are independent but no longer identically distributed. The achievable diversity for a given multiplexing gain in independent complex Gaussian channels was shown in [9] to be no different than when the entries are i.i.d. complex Gaussian as $P_{a v} \rightarrow \infty$. Thus, our result holds even when the average power constraint per transmit antenna is relaxed.

\section{NumERICAL RESUlTS}

1) Asymmetric D-MG Tradeoff: Consider a MAC with two users having $m_{1}=2, m_{2}=3$ transmit antennas and $n=4$ receive antennas. The capacity region is given as [12],

$$
\left\{\left(r_{1}, r_{2}\right): r_{1} \leq 2, r_{2} \leq 3 \text { and } r_{1}+r_{2} \leq 4\right\},
$$

where $r_{i}=\min \left\{m_{i}, n\right\}$. Thus, given any rate pair in (16), the D-MG tradeoff region, $d_{1}^{p c}\left(r_{1}, r_{2}\right)$, for user 1 with 1 bit of feedback $(L=2)$ is computed using (11) as,

$$
d_{1}^{p c}\left(r_{1}, r_{2}\right)=\min \left\{d_{m_{1}, n, L}^{p c}\left(r_{1}\right), d_{m_{1}+m_{2}, n, L}^{p c}\left(r_{1}+r_{2}\right)\right\} .
$$

This maximal diversity, $d_{1}^{p c}\left(r_{1}, r_{2}\right)$, is plotted as a function of $r_{1}$ for $r_{2}=\{0,1,2,3\}$ in Fig. 3(a). The discontinuities at integer multiplexing gains in the Fig. 3(a) is an artefact of switching eigenvalues [6]. From the figure, we can see that for $r_{2} \in(0,1]$, user 1 attains single user tradeoff i.e., $d_{1}^{p c}\left(r_{1}, r_{2}\right)=d_{m_{1}, n, L}^{p c}\left(r_{1}\right)$. As $r_{2}$ increases beyond one, the maximum achievable D-MG tradeoff for user 1 reduces from the single user tradeoff curve due to the dominating effect of the sum-rate diversity, $d_{m_{1}+m_{2}, n, L}^{p c}\left(r_{1}+r_{2}\right)$. However, we can increase the per user diversity, by simply increasing the number of bits of feedback since the achievable diversity tends to infinity as $B \rightarrow \infty$.

2) Symmetric $D-M G$ tradeoff: Consider a two user MAC with $m_{1}=m_{2}=m=3$ transmit antennas, $n=4$ receive antennas and $B=1$ bit of feedback. When both users transmit at same rate, i.e., $r_{1}=r_{2}=r$, the D-MG tradeoff for both users will be the same and is computed using (11) as,

$$
d^{p c}(r, r)=\left\{\begin{array}{c}
d_{m, n, L}^{p c}(r) \quad r \leq r^{\prime} \\
d_{2 m, n, L}^{p c}(2 r) \quad r \geq r^{\prime}
\end{array}\right.
$$




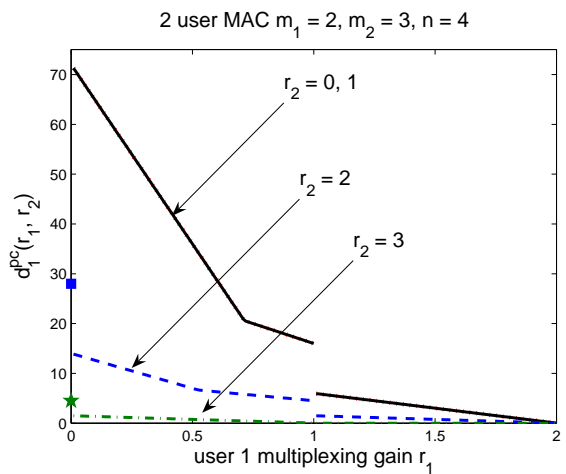

(a)

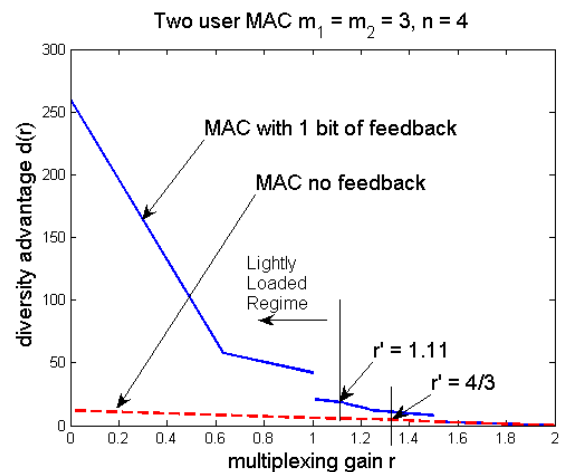

(b)

Fig. 3. (a) The D-MG tradeoff for user $1, d_{1}^{p c}\left(r_{1}, r_{2}\right)$ as a function of $r_{1}$ at fixed values of $r_{2}$. The points, $\mathbf{\square}$ and $\star$ indicate the values of $d_{1}^{p c}\left(0, r_{2}\right)$ when $r_{2}=2$ and 3 respectively. (b) The symmetric D-MG tradeoff.

where $r^{\prime}$ is the intersection point of the curves, $d_{m, n, L}^{p c}(r)$ and $d_{2 m, n, L}^{p c}(2 r)$. For all $r \leq r^{\prime}$, single user performance is achieved by each user in the MAC and is called lightly loaded regime. In the lightly loaded regime, it is as if $B$ bits each are transmitted to user 1 and 2 , when in fact just $B$ bits are broadcast to all users. For $r \geq r^{\prime}$, it is as though the two users are pooled together i.e., like a single user with $2 m$ antennas and multiplexing gain $2 r$; this regime is said to be heavily loaded. The D-MG tradeoff with PC described in (17) is plotted in Fig. 3(b). For comparison, we also plot the optimal D-MG tradeoff with no PC [13]. The threshold $r^{\prime}$ that differentiates the lightly and heavily loaded regimes is evaluated numerically for PC-MAC while for the MAC with no CSIT, $r^{\prime}=n /(K+1)$ [13].

3) Power Control vs. Opportunistic User selection: Opportunistic user selection is shown [14] to be sum-rate optimal for a SISO MAC ${ }^{1}$. In each coherence internal, the BS has perfect channel knowledge $h_{i} \forall i$, and picks the user with the maximum channel gain, $\max _{i}\left|h_{i}\right|^{2}$ for transmission over the entire bandwidth. This choice is communicated to the users with $B=\log _{2} K$ bits of feedback in each coherence interval. The symmetric D-MG tradeoff for a MAC with user selection is shown to be [8],

$$
d(r)=K(1-K r)^{+} .
$$

In contrast, $\mathrm{PC}$ in a SISO MAC requires that power be allocated based on careful eigenvalue selection to ensure that all users get sufficient power to minimize outage. In a SISO MAC, the eigenvalue of the concatenated matrices of all subsets, $S$, is, $\left\{\sum_{S}\left|h_{i}\right|^{2}, \forall S \subseteq\{1, \ldots, K\}\right\}$. To ensure that the transmit power is sufficient for all users to support their desired rate, we quantize $\min _{i}\left|h_{i}\right|^{2}$. For a fair comparison, we assume that $\min _{i}\left|h_{i}\right|^{2}$ is quantized to $B=\log _{2} K$ bits. Then, the symmetric D-MG tradeoff with PC is computed using (11) evaluates to (18). Thus, the PC and user selection strategies have D-MG tradeoff.

${ }^{1}$ For a MIMO MAC, user selection does not use all degrees of freedom and is sub-optimal [15].

\section{CONCLUSIONS}

We characterized the achievable diversity using PC with $B$ bits of feedback for any given rate vector in a MIMO MAC. We also showed that all users can achieve their maximum diversity gains simultaneously. Further, we showed the DMG equivalence between the proposed PC and user selection strategy in a SISO MAC. Future work should focus on deriving the optimal D-MG tradeoff with finite rate feedback.

\section{REFERENCES}

[1] K. Premkumar et al., "Exponential diversity achieving spatio-temporal power allocation scheme for MIMO fading channels," in Proc. IEEE Int. Sym. Inf. Th., Chicago, IL, June 2004.

[2] G. Caire et al., "Optimum power control over fading channels," in IEEE Trans. Inf. Th., vol. 45, no. 5, July 1999, pp. 1468-1489.

[3] S. K. Jayaweera and H. V. Poor, "Capacity of multiple-antenna systems with both receiver and transmitter channel state information," in IEEE Trans. Inf. Th., vol. 49, no. 10, Oct 2003, pp. $2697-2709$.

[4] L. Zheng and D. Tse, "Diversity and multiplexing: A fundamental tradeoff in multiple-antenna channels," in IEEE Trans. Inf. Th., vol. 49 no. 5, May 2003, pp. $1073-1096$.

[5] L. G-Ordóñez et al., "Diversity and multiplexing tradeoff in multiple beamforming in MIMO channel," in Proc. IEEE Int. Sym. Inf. Th., Adelaide, Australia, Sep 2005.

[6] A. Khoshnevis and A. Sabharwal, "Achievable diversity and multiplexing in multiple antenna systems with quantized power control," in Proc. of ICC05, Seoul, South Korea, May 2005.

[7] M. Kountouris et al., "Multiuser diversity-multiplexing tradeoff in MIMO broadcast channels with limited feedback," in Proc. of 40th Asilomar Conf., Pacific Grove, CA, Oct-Nov 2006.

[8] K. Josiam and D. Rajan, "Multiuser diversity in wireless networks: A diversity-multiplexing perspective," in Proc. of 41st CISS, Baltimore, MD, Mar 2007.

[9] L. Zhao et al., "Diversity and multiplexing tradeoff in general fading channels," in Proc. of 40th CISS, Princeton, NJ, Mar 2006.

[10] K. Josiam et al., "Optimal diversity multiplexing tradeoff region in asymmetric multiple access channels," in to appear in IEEE VTCFall'07, Baltimore, MD, 2007.

[11] E. Biglieri, G. Caire, and G. Taricco, "Limiting performance of blockfading channels with multiple antennas," in IEEE Trans. Inf. Th., vol. 47, no. 4, May 2001, pp. 1273 - 1289.

[12] D. Tse and P. Viswanath, Fundamentals of Wireless Communication. Cambridge University Press, 2005.

[13] D. Tse et al., "Diversity-multiplexing tradeoff in multiple-access channels," in IEEE Trans. Inf. Th., vol. 50 no.9, Sep 2004, pp. 1859-1874.

[14] R. Knopp and P. Humblet, "Information capacity and power control in single-cell multiuser communications," in Proc. of IEEE ICC'95, Seattle, WA, June 1995, pp. 331-335.

[15] W. Yu and W. Rhee, "Degrees of freedom in wireless multiuser spatial multiplex systems with multiple antennas," in IEEE Trans. Wireless Comm., vol. 54, no. 10, October 2006, pp. 1747 - 1753. 